\title{
gb4gv: A genome browser for geminivirus
}

\author{
Eric S Ho ${ }^{\text {Corresp., }}{ }^{1,2}{ }^{\text {, }}$ Catherine M Newsom-Stewart ${ }^{1}$, Lysa Diarra $^{1}$, Caroline S McCauley $^{1}$ \\ 1 Department of Biology, Lafayette College, Easton, Pennsylvania, United States \\ 2 Department of Computer Science, Lafayette College, Easton, Pennsylvania, United States \\ Corresponding Author: Eric S Ho \\ Email address: hoe@lafayette.edu
}

Background: Geminiviruses (family Geminiviridae) are prevalent plant viruses that imperil agriculture globally, causing serious damage to the livelihood of farmers, particularly in developing countries. The virus evolves rapidly, attributing to its singlestranded genome propensity, resulting in worldwide circulation of diverse and viable genomes. Genomics is a prominent approach taken by researchers in elucidating the infectious mechanism of the virus. Currently, NCBI Viral Genome website is a popular repository of viral genomes that conveniently provides researchers a centralized data source of genomic information. However, unlike the genome of living organisms, viral genomes most often maintain peculiar characteristics that fit into no single genome architecture. By imposing a unified annotation scheme on the myriad of viral genomes may downplay their hallmark features. For example, the viron of begomoviruses prevailing in America encapsulates two similar-sized circular DNA components and both are required for systemic infection of plants. But, the bipartite components are kept separately in NCBI as individual genomes with no explicit association in linking them. Thus, our goal is to build a comprehensive Geminivirus genomics database, namely gb4gv, that not only preserves genomic characteristics of the virus, but also supplements biologically relevant annotations that help to interrogate this virus e.g. the targeted host, putative iterons, siRNA targets etc. Methods: We have employed manual and automatic methods to curate 508 genomes from four major genera of Geminiviridae, and 161 associated satellites obtained from NCBI RefSeq and PubMed databases. Results: These data are available for free access without registration from our website. Besides genomic content, our website provides visualization capability inherited from UCSC Genome Browser. Discussion: With the genomic information readily accessible, we hope that our database will inspire researchers in gaining a better understanding of the incredible degree of diversity of these viruses, and of the complex relationships within and between the different genera in the Geminiviridae. Availability and Implementation: Database URL: http://gb4gv.lafayette.edu . 


\section{1 gb4gv: A Genome Browser for Geminivirus}

2 Eric S. Ho ${ }^{1,2}$, Catherine M. Newsom-Stewart ${ }^{1}$, Lysa Diarra ${ }^{1}$, and Caroline S. McCauley ${ }^{1}$

$3{ }^{1}$ Department of Biology, ${ }^{2}$ Department of Computer Science, Lafayette College, Easton,

4 Pennsylvania, 18042, United States.

5 Corresponding author: Eric S. Ho

6 Email address: hoe@lafayette.edu

\section{Abstract}

8 Background: Geminiviruses (family Geminiviridae) are prevalent plant viruses that imperil

9 agriculture globally, causing serious damage to the livelihood of farmers, particularly in

10 developing countries. The virus evolves rapidly, attributing to its single-stranded genome

11 propensity, resulting in worldwide circulation of diverse and viable genomes. Genomics is a

12 prominent approach taken by researchers in elucidating the infectious mechanism of the

13 virus. Currently, NCBI Viral Genome website is a popular repository of viral genomes that

14 conveniently provides researchers a centralized data source of genomic information.

15 However, unlike the genome of living organisms, viral genomes most often maintain

16 peculiar characteristics that fit into no single genome architecture. By imposing a unified

17 annotation scheme on the myriad of viral genomes may downplay their hallmark features.

18 For example, the viron of begomoviruses prevailing in America encapsulates two similar-

19 sized circular DNA components and both are required for systemic infection of plants. But,

20 the bipartite components are kept separately in NCBI as individual genomes with no

21 explicit association in linking them. Thus, our goal is to build a comprehensive Geminivirus

22 genomics database, namely gb4gv, that not only preserves genomic characteristics of the 
23 virus, but also supplements biologically relevant annotations that help to interrogate this

24 virus e.g. the targeted host, putative iterons, siRNA targets etc.

25 Methods: We have employed manual and automatic methods to curate 508 genomes from

26 four major genera of Geminiviridae, and 161 associated satellites obtained from NCBI

27 RefSeq and PubMed databases.

28 Results: These data are available for free access without registration from our website.

29 Besides genomic content, our website provides visualization capability inherited from

30 UCSC Genome Browser.

31 Discussion: With the genomic information readily accessible, we hope that our database

32 will inspire researchers in gaining a better understanding of the incredible degree of

33 diversity of these viruses, and of the complex relationships within and between the

34 different genera in the Geminiviridae.

35 Availability and Implementation: Database URL: $\underline{\text { http://gb4gv.lafayette.edu. }}$

\section{Introduction}

Geminiviruses (family Geminiviridae) have emerged as one of the most prevalent and problematic plant pathogens especially in developing countries (Sattar et al. 2013;

Scholthof et al. 2011; Shepherd et al. 2010). In terms of diversity, they have become the largest group of plant viruses to exist today. It posts significant threat both socially and economically as geminiviruses are the most destructive pathogens in subsistence agriculture like beans, cotton, maize, sweet potato, and tomato (Jeske 2009; Sattar et al.

44 2013; Scholthof et al. 2011; Shepherd et al. 2010). The economic impact of geminivirus

45 infection can be seen across the globe. Annual economic loss is estimated to be US\$1.9-2.7 
46 billion in East and Central Africa. Maize streak virus alone has caused hundreds of millions

47 of loss in food crops per year (Shepherd et al. 2010).

Geminiviruses often infect plants as complexes: a mixture of viral isolates identified as distinct species, as well as DNA satellites. Moreover, they are able to undergo mutation, recombination and reassortment both frequently and rapidly. Together, these factors

51 increase the diversity and capabilities of the family, allowing them to invade new hosts and

52 new environments without complication. In order to prevent geminiviruses from becoming even more of a threat to our growing human population, it is critical that scientists are able

54 to better understand the genomic sequences of these viruses. Geminiviruses rely heavily on 55 their host's cellular machinery so having a greater knowledge of their genetic makeup will 56 allow scientists to formulate biotechnological means to help plants fight their attackers 57 successfully.

Geminiviruses comprise a family of plant viruses that exist in the form of twinned icosahedral particles holding small, circular, single stranded deoxyribonucleic acid (ssDNA) genomes. The ssDNA genome structure enables it to evolve at high rate comparable to RNA viruses (Duffy et al. 2008). The viral genome encodes only 5-7 proteins, making geminiviruses one of the smallest virus types known to scientists today. Within Geminiviridae, seven genera are currently recognized by The International Committee on

64 Taxonomy of Viruses (ICTV): Becurtovirus, Begomovirus (the one with the largest number of species), Curtovirus, Eragrovirus, Mastrevirus, Topocuvirus, and Turncurtovirus. 
69 alpha- or betasatellite, while bipartite genomes consist of separated DNA-A and DNA-B 70 components of similar size.

71 National Center for Biotechnology Information (NCBI) designates a separate

72 website to host viral genomes (NCBI Viral Genomes). Its collection includes almost all

73 known viruses in the world, making it one of the most comprehensive resources for

74 studying viral genomics. Viral genomes are formatted in standard GenBank record

75 (GenBank Record) exactly like other living organisms. However, genome architectures of

76 viruses exhibit significant difference from living organisms. For instance, virions of

77 bipartite begomoviruses encapsulate two circular, ssDNA components in which the two

78 components are required for infectivity. But such critical association between the two

79 components is often missing from NCBI Viral Genome database. Moreover, vital

80 information about the virus such as location where it was found, targeted hosts, etc. are not

81 searchable attributes, limiting the utility of the database. These are the reasons that we

82 have undertaken this project in providing researchers a comprehensive, up-to-date, and

83 integrated environment at their fingertips. The database we built rests on the software

84 architecture of UCSC Genome Browser website (Kent et al. 2002; UCSC Genome Browser)

85 as such we named our database gb4gv, which stands for Genome Browser for Geminivirus.

86 For clarity, we reserve "UCSC Genome Browser" to refer to the website itself (UCSC

87 Genome Browser), and "Genome Browser" to mean the software that supports the website.

88 Genome Browser was chosen because of its versatility in visualizing genomes, richness in

89 built-in functions, flexibility in incorporating annotations, and software robustness in

90 handling large volume of requests - 872,000 requests per day on average (UCSC GB

91 Statistics). Although Genome Browser offers these benefits, its original design gears mainly 
92 toward eukaryotes. In order to unleash the power of Genome Browser, we have made

93 substantial effort in modeling geminivirus genomes into a structure that can take full

94 advantage of its functionalities. gb4gv can be accessed, without registration requirements,

95 from here: http://gb4gv.lafayette.edu. Users can make use of the built-in functions

96 provided through our website to download genomes or sequences of interested regions

97 freely.

98 Materials \& Methods

99 Compilation of Geminivirus Genomes 


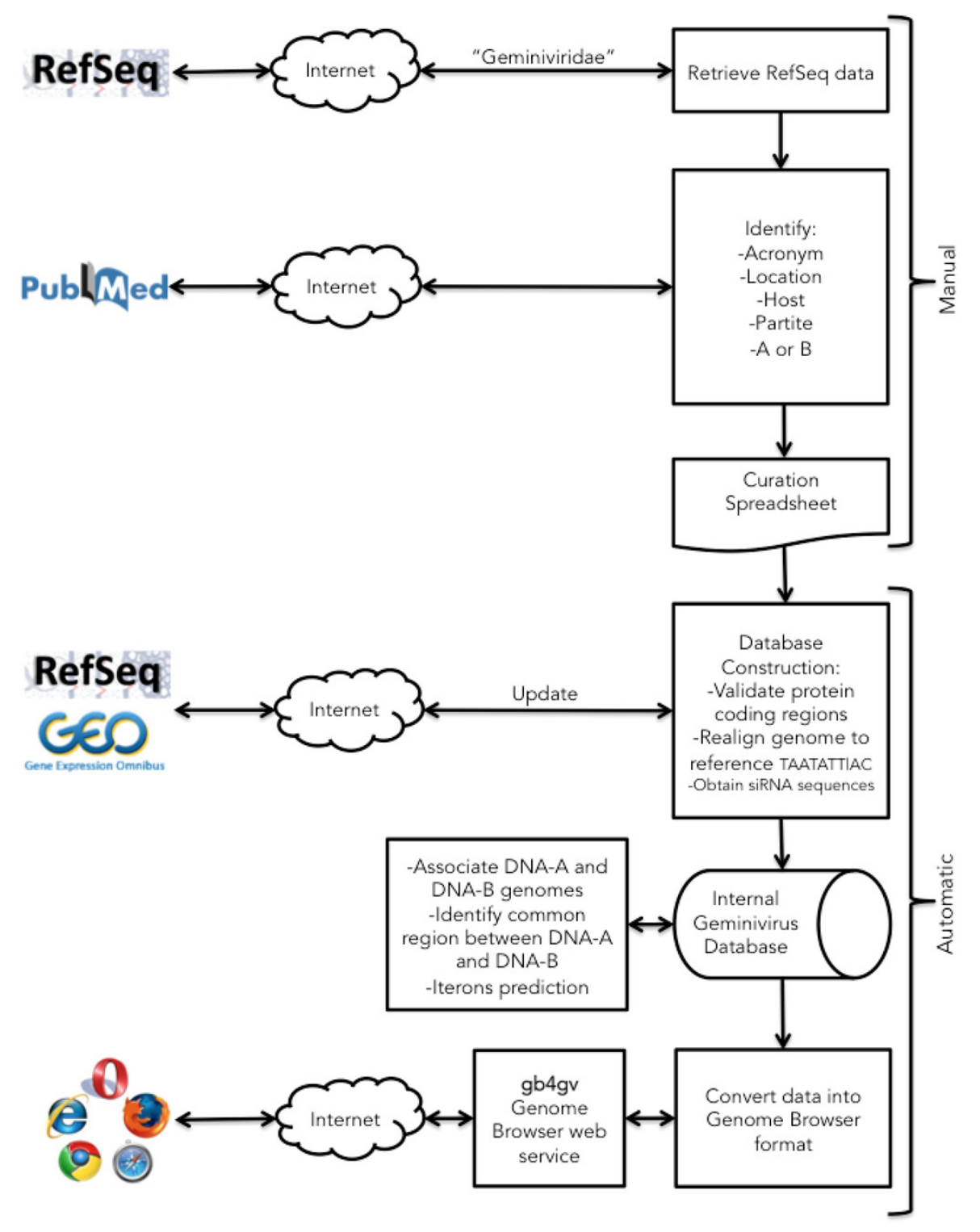

101 Figure 1 Project workflow of the semi-automatic annotation process. Our workflow begins 102 with a manual process to identify information generally not documented in the GenBank record such as the acronym of the virus, location, infecting host, monopartite or bipartite genome, and genomes association for bipartite virus. This information is passed to a downstream automatic process that integrates them with other sources. The automatic procedure parses GenBank entries from RefSeq database for genomic information of geminiviruses including the accession numbers, genomic sequences, genes, viral proteins, and taxonomy ID. siRNAs from host plants that fight against viral infection were obtained from NCBI GEO database. In the last step, geminivirus information is formatted into UCSC 
112 Figure 1 above summarizes the semi-automatic annotation workflow designed for this

113 project. The primary source of our data originated from NCBI RefSeq database (NCBI

114 RefSeq) because redundant genomes were purged. But we also cross-referenced our data

115 with the ICTV Master Species List 2015 v1 obtained from ICTV (ICTV 2015). We had

116 identified 700 RefSeq entries that comprised of 529 distinct geminiviruses. Note that DNA-

117 A and DNA-B of a bipartite begomovirus are kept in separate entries in RefSeq.

118 Begomovirus occupies the largest genus of the family, followed by Mastrevirus, and

119 Curtovirus. Other genera were found sporadically including two becurtoviruses, one

120 topocuvirus, one eragrovirus, and one turncurtovirus, while genera of ten entries remain

121 unknown. Here we had decided to incorporate only genera that represent major

122 Geminiviridae genera i.e. Begomovirus, Curtovirus, and Mastrevirus into gb4gv. As a result,

123 genomes of 514 geminiviruses representing 97\% of the Geminiviridae found in NCBI were

124 considered for further review. We will regularly assess the need to include other minor

125 genera into our database if more samples from them are discovered in the future.

126 Besides the main genomes, ancillary alphasatellites and betasatellites are often isolated

127 together with monopartite begomoviruses (Xie et al. 2010) and they are found to play

128 essential roles in boosting host's symptoms and viral movement (Briddon et al. 2001;

129 Saunders et al. 2004; Zhou et al. 2003). We had identified and reviewed 66 and 105

130 alphasatellite and betasatellite genomes, respectively, from NCBI.

131 Meta information or attributes such as the geographical location of the virus are

132 important to understand the virulence of the virus but it is not always available in genome

133 database. Therefore we manually searched for additional information about these viruses

134 from existing literature. In particular, we focused on identifying or reconfirming the 
135 location where they were collected, the hosts they infected, their acronyms, monopartite or

136 bipartite genome, and the counterpart genome in case of bipartite. Importantly, we have

137 made these attributes searchable in our database.

138 Following the manual process is the automatic annotation process. In this step, NCBI

139 RefSeq entries belonging to Geminivirus were parsed to ensure that each entry satisfies the

140 following two criteria:

141 1. Every geminivirus and geminivirus-associated DNA satellite genomes must possess the

142 iconic structurally conserved element (SCE), which is the genomic landmark of

143 geminiviruses including satellites. The canonical structure of the SCE is TAATATT|AC,

144 where "|" stands for the cleavage site targeted by the viral replication protein in the

145 initial step of DNA replication (Gutierrez 1999; Jeske et al. 2001; Pilartz \& Jeske 2003).

146 The prevalent SCE sequence of alphasatellite is TAGTATT |AC, which varies slightly

147 from the canonical SCE sequence. Nonetheless, owing to either DNA sequencing errors

148 or random mutations, the 5' side of the SCE of some viruses may deviate slightly (less

149 than one nucleotide) from the canonical form from above. To accommodate such

150 minutiae, we tolerated entries with up to one mismatch from TAATATT. Genomes failed

151 to meet this criterion were excluded from gb4gv.

152 2. Besides genomes, gb4gv also keeps individual viral proteins if they satisfy our quality

153 checking. The coding region (CDS) of a gene defined in a RefSeq entry must be

154 translated exactly into the stated peptide in the RefSeq entry. Genes failed this criterion

155 were excluded from our database. But genomes containing erroneous CDS were still

156 kept in the database. 
158 Through our tandem manual and automatic annotations, 6 out of 514 RefSeq entries of

159 geminiviruses failed the validation process stated above, resulting in 508 genomes being

160 selected into our database. For satellite genomes, 7 out of 66 alphasatellites and 3 out of

161105 betasatellites failed our validation. Table 1 below categorizes all the genomes accepted

162 into our database by genus, number of genomes per virus, and geographical origin. The

163 aforementioned annotation information can be downloaded from our website in tab-

164 separated format (http://gb4gv.lafayette.edu/downloads.html).

165 Table 1. A summary of genomes stored in gb4gv. The numbers inside the parentheses

166 denote the numbers of genomes. The lower part of the table categorizes begomoviruses

167 further by origin and the number of genomes per virus.

Geminiviridae (508) $\quad$ Satellite (161)

Begomovirus (470)

\begin{tabular}{cccc|cc} 
Curtovirus & Mastrevirus & DNA-A & DNA-B & Alphasatellite & Betasatellite \\
\hline 5 & 34 & 338 & 132 & 59 & 102 \\
\hline
\end{tabular}

168

Begomovirus (338)

Old World (216) New World (119) N N Unknown origin (2)

\begin{tabular}{cccccccc}
\hline Monopartite & Bipartite & Unknown & Monopartite & Bipartite & Unknown & Monopartite & Bipartite \\
100 & 44 & 72 & 12 & 95 & 13 & 1 & 1 \\
\hline
\end{tabular}

169

170 Small Interfering RNAs

171 A key aspect of gb4gv is to inspire researchers to formulate insightful strategies that can be

172 used to eradicate the propagation of geminiviruses. Therefore, studying the immune

173 response launched by infected plant is a promising research direction. Thus, we had

174 downloaded datasets from two small interfering RNAs studies deposited in NCBI GEO 
175 database (Gene Expression Omnibus): GSM425427, and GSE26368. siRNA sequences were

176 mapped to the genomes of begomovirus and betasatellite through a customized Python

177 script. Mapping tolerated up to two mismatches in internal positions without gaps. A

178 Genome Browser annotation track is designated for each sample, which can be found under

179 "Mapping and Sequencing" section of each virus. In Begomovirus or betasatellite, six siRNA 180 tracks were configured.

181

182 Standardization of Circular Genomes

183 Like many other genomic databases such as NCBI RefSeq and UCSC Genome Browser, 184 circular genomes are linearized. Instead of opening the circular genome at arbitrary sites, 185 circular genomes were opened at the biological cleavage site at the SCE. A benefit of 186 standardizing the opening site is to facilitate syntenic analysis (to be discussed in Multiz 187 section below). Under the standardized linearization scheme, a genome always begins with 188 'AC' and terminates with 'TAATATT' at the 5' and 3 ' termini, respectively. Thereby we 189 standardized all genomes obtained from RefSeq. Genomes not conforming to this standard 190 were shifted until they met the above criterion. Out of 669 accepted genomes in gb4gv, 191 surprisingly, $112(17 \%)$ of them required this adjustment.

193 Data Models

194 Genome Browser was originally designed to visualize mammalian genomes (Kent et al. 195 2002). It was later enhanced to host non-mammalian animals e.g. C. elegans, and then 196 unicellular organisms such as yeast. Ebola genome is the first and remains to be the only 197 viral genome available in UCSC Genome Browser at present. This historical background 
198 reveals that the data model of Genome Browser is geared toward the display of

199 chromosomes of a species. Such data model serves well with living organisms but it poses

200 two challenges in configuring Genome Browser for geminivirus genomes:

201 1. The genus Begomovirus is known to be diverse (Brown et al. 2015) with over 300 DNA

202 components being identified by us. If we were to coerce the existing data model to

203 begomoviruses, 300 databases are needed, leading to a huge species tree in the home

204 page, hampering website performance, and prohibiting data browsing. To circumvent

205 this, we modeled each viral genus as an organismal species, and the array of viral

206 species of a genus as chromosomes of an organism. Based on this workaround, gb4gv

207 consists of five databases (a database per genus including one for each satellite

208 although, in biological terms, satellite is not considered a genus): Begomovirus,

209 Mastrevirus, Curtovirus, alphasatellite, and betasatellite.

210 2. A special configuration is needed to establish the association between the bipartite DNA

211 components (DNA-A and DNA-B) of a begomovirus. In gb4gv, DNA-A and DNA-B were

212 treated as two separate chromosomes. The coupling of DNA-A and DNA-B components

213 of a bipartite begomovirus can only be achieved manually as their RefSeq accession

214 numbers reflect no information about their relationship. In order to facilitate users to

215 associate them easily, a viral species in our database is uniquely referenced by an

216 acronym, e.g. AbMBV is the reference of Abutilon mosaic Brazil virus. But the two DNA

217 components of a bipartite begomovirus will become indistinguishable under this

218 scheme. Thus, we suffix the acronym of a bipartite virus by ".A" and ".B". E.g. the DNA-A

219 and DNA-B of the virus AbMBV can be found effortlessly through AbMBV.A and

220 AbMBV.B, respectively. An advantage of using an acronym as the key to retrieve a virus 
221 is to release the burden of users to pull up the accession number of the virus as most

222 people can remember the acronym rather than the arbitrary accession number.

223 Moreover, we recognize that some viruses are referenced by multiple acronyms

224 without a consensus. To accommodate such variability our database maintains a list of

225 searchable aliases for every virus, e.g. Tomato leaf curl New Delhi virus can be

226 identified by either TolCNDV or ToLCV_India.

227

228 Common Region Identification in Bipartite Begomoviruses

229 The bipartite genomes of a begomovirus share a highly similar, non-coding segment

230 flanking the SCE "TAATATTAC". This segment is colloquially named the common region

231 (CR). CRs serve a crucial role in viral DNA replication. Studies had shown that the 5' side of

232 CRs contain replication protein binding sites (Orozco \& Hanley-Bowdoin 1996). Thus, CRs

233 harbor vital regulatory signals that influence the replication and the coupling of the

234 bipartite genomes for begomoviruses. Understanding viral replication is fundamental to

235 combat viral infection. Thus, we undertook the task to predict CRs in bipartite

236 begomoviruses. Based on the manual annotation we did, DNA-A and DNA-B components of

237 a begomovirus were paired up. We extracted the non-coding region, also known as the long

238 intergenic region (LIR), between REP and CP genes in the DNA-A or between NSP and MP

239 genes in the DNA-B. In the next step, we further reduced the LIR into an 809-bp segment,

240 which consisted of a 400-bp segment upstream and downstream of the SCE from the DNA-

241 A and DNA-B. In Figure 2 below, two 809-bp segments were aligned by MUSCLE (Edgar

242 2004) as shown: 


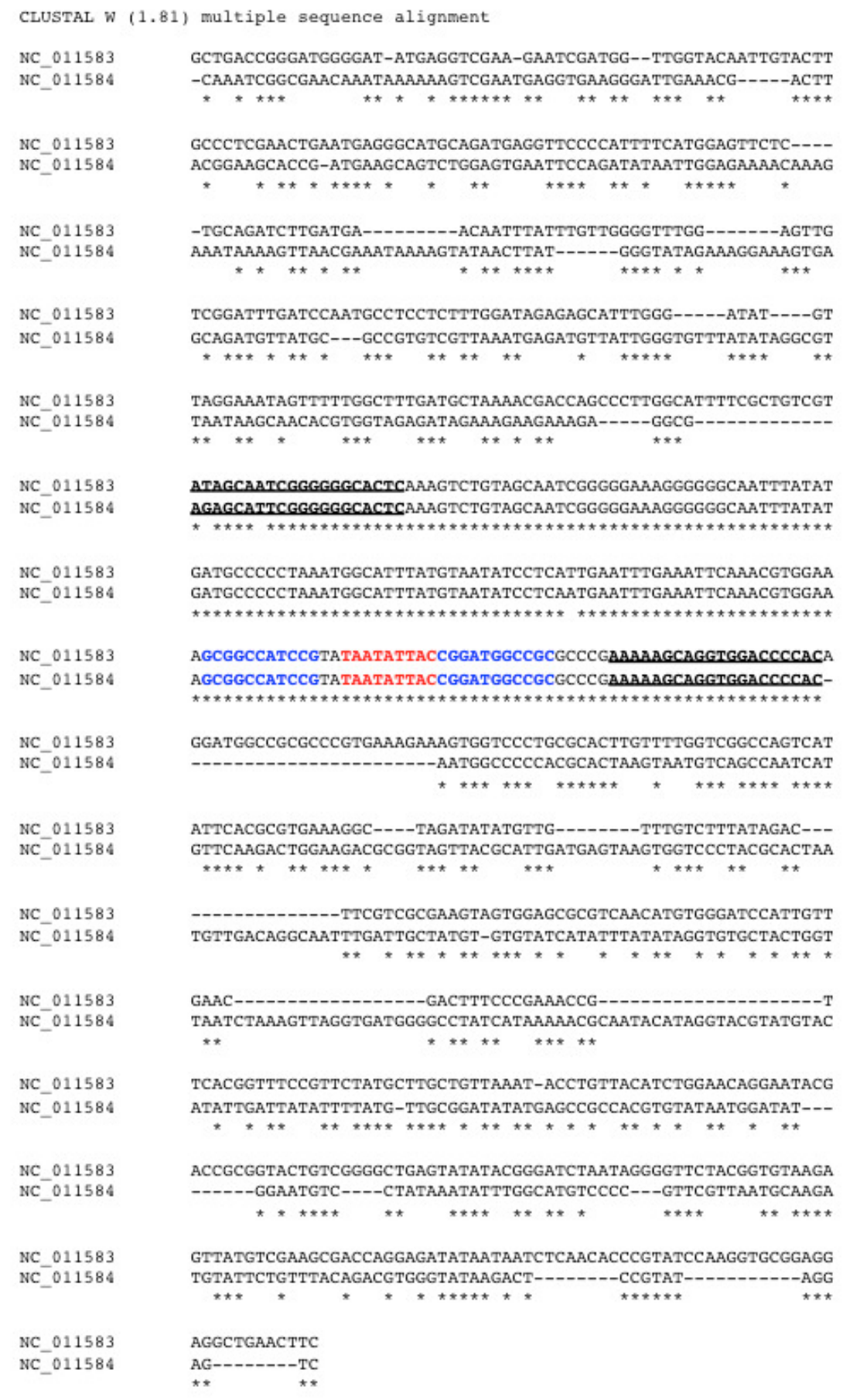

Figure 2. Identification of common region (CR) shared between DNA-A and DNA-B of the Old World bipartite East African cassava mosaic Kenya virus (EACMKV). The invariant SCEs are highlighted in red. The inverted repeats constituted the stem of the hairpin structure are highlighted in blue. The two underlined regions indicate the 5' and 3' 
251 In this example, the two segments were extracted from DNA-A (NC_011583) and

252 DNA-B (NC_011584) of the Old World bipartite East African cassava mosaic Kenya virus

253 (EACMKV) and they were aligned. A 20-bp sliding window was used to scan the alignment

254 base-by-base bilaterally starting from the SCE. Scanning halts when the percentage of

255 sequence identity within the window drops below $80 \%$, an adjustable parameter. The

256 halting locations (the underlined regions in Figure 2) are taken as the 5' and 3' termini of

257 the common region.

258 The average size of a CR was found to be 212 bps (including the SCE) in which the $5^{\prime}$

259 arm, the left segment of the SCE, is usually longer than the 3' arm with an average size of

260150 bps. The longest CR is $417-419$ bps long that belongs to Indian cassava mosaic virus

261 (NC_001932/NC_001933). Whereas Abutilon mosaic Brazil virus (NC_016574/NC_016577)

262 was found to possess the shortest CR, which is 63-67 bps long. Also note that the two

263 approximately 10 bps segments juxtaposing the SCE constitute the stem part of the hairpin

264 structure (Figure 2).

265

266

Putative Iterons and TATA Box Sites

267 One of the cis-regulatory signals harboring in CR is iterative elements, also known as

268 iterons (Arguello-Astorga et al. 1994; Arguello-Astorga \& Ruiz-Medrano 2001; Sanz-Burgos

$269 \&$ Gutierrez 1998). A distinct feature of iterons is the presence of direct or inverted

270 sequence repeats. The following rules were applied to predict iterons in viral genomes:

271 1. They are located in the 5' side of the LIR i.e. from the beginning of the first gene on the

272 complementary strand up to, but excluding, the SCE 
273 2. The minimum length of an iteron is $9 \mathrm{bps}$. There is no restriction on the maximum $274 \quad$ length

2753 . The pair of repeats identified differ by at most one base

276 4. Repeats could be direct or inverted

277 5. Its location is no more than $100 \mathrm{bps}$ from a putative TATA box, if any

278 6. At least one of the twelve iteron core motifs is present (Arguello-Astorga \& RuizMedrano 2001): GGAGN, GGTAV, GGGGW, GGTAV, GGKGT, GGKGG, GGGGG, GGGGA, GGGTM, GGCGT, GGWGT, and TGGTGTCC.

281

282

As the TATA box sites and iterons work cooperatively to regulate replication, we also 283 identified putative TATA box sites. The consensus sequence of a TATA box is defined as 284 “TATA", followed by any number of “TA" or "AA" repeat (Bernard et al. 2010; Patikoglou et

285

286

287 288 289 290

291 292 293 294 295

al. 1999). We developed a Python script to scan for iterons and TATA box sites in every geminivirus genome. Based on the above criteria, 5,142 iterons and TATA box sites were predicted from 669 components and genomes. Results can be visualized in gb4gv by activating the "Iterons and TATA" annotation track.

\section{Multiz Track}

Genomes of various species within a genus share similarities and differences. Since we have standardized the opening site of the viral circular genomes, a genus-wide syntenic analysis becomes possible. Such comparative view helps to uncover conserved and diverse genomic regions among species. We used the threaded blockset aligner (TBA) (Blanchette et al. 2004) to generate a dynamic multiple sequence alignments of all species in a genus. 
296 Unlike other multiple sequence alignment programs of which a sequence from the sample

297 is dedicated to be the reference of the alignment, TBA produces a multiple sequence

298 alignment dynamically based upon the genome being selected for viewing in the Genome

299 Browser. This unique feature enables gb4gv to generate a graphical representation

300 regarding genome conservation among different species with respect to the current 301 queried genome.

302 TBA requires two mandatory inputs: a set of genomic sequences, and a phylogenetic

303 tree defining the evolutionary relationship of the input genomes. We used multiple

304 sequence alignment program MUSCLE (Edgar 2004) to build phylogenetic trees, followed

305 by maximum likelihood tree building PHYLM (Felsenstein 2005) equipped in MEGA7

306 (Kumar et al. 2016). The output phylogenetic tree was in NEWICK format. Based on the

307 genomic sequences and the phylogenetic tree, TBA generated the threaded blockset

308 alignment. The alignment was loaded to a MySQL database referenced by Genome Browser. 309

UniProt/SwissProt Annotations

Protein domain information was overlaid on viral proteins in gb4gv. Reviewed

312 Swiss-Prot annotations were downloaded from UniProt website in XML format

313 (UniProtKB). Viral taxonomy IDs served as the key to retrieve protein domain information

314 from the Swiss-Prot annotations. Sequence of the protein domains identified in the search

315 process was mapped to the genomes by BLAT (Kent 2002).

316

317 Genome Browser 
318 Version 334 of the Genome Browser was used to build gb4gv. The software was

319 downloaded from the UCSC Genome Browser website (UCSC Admin) and installed in our

320 24-core Linux server running on Centos OS 6.8, Apache 2.2, and MySQL server 5.5.50.

321

\section{Results}

323 The web interface of gb4gv is organized in a hierarchy consisting of three levels. The

324 highest level presents all the genera of Geminiviridae maintained in gb4gv including two

325 satellites despite they are considered as genera (Figure 3A). The middle level displays

326 information about the genome of an individual virus and corresponding annotation tracks

327 (Figure 3B). Detailed information about a particular annotation e.g. a gene, a protein or a

328 specific genomic sequence, is presented at the lowest level (Figure 3C) 
A LAFAYETE! Genome Browser Gateway

A Genomes Genome Browser Tools Downloads Help About Us

Browse/Select Species

SPEcies search

Enter species or common name

REPRESENTED SPECIES

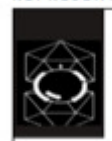

Alphasatellites

Betasatellites

Begomovirus

Curtovirus

Mastrevirus

\section{Find Position}

Begomovirus Assembly

July 2016

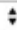

Position/Search Term

Enter position, gene symbol or search terms

Current position: TYLCV.A Q

\section{Begomovirus Genome Browser - begVir1 assembly}

Begomovirus constitutes the largest genus in the Geminiviridae family. It infects

monocotyledons and dicotyledons including staple food worldwide, causing severe damages to agriculture especially in developing countries. Begomovirus is further classified into

monopartite and bipartite depending on whether the virion encapsulates one or two circular

ssDNA genomes. The genome that shares similar gene architecture between monopartite and

bipartite viruses is commonly called DNA.A. And the second genome of bipartite viruses is

called DNA-B. Our database contains 139 and 113 bipartite and monopartite begomoviruses.

respectively. DNA-A and DNA-B of a virus are labeled by its viral abbreviation suffixed with .A

and .B, respectively. E.g. Genomes of Melochia yellow mosaic virus are represented by

MeIYMVA and MeIYMVB in our database. For monopartite begomoviruses, their genomes are always sur

e.g. genome of Tomato leaf curl Hainan virus is represented by ToLCHaiVA.

GB4GV Genome Browser on Begomovirus July 2016 Assembly (begVir1)

B



TYLCVNV.A:1-2,745 2,745 bp. Ener posinon or wewch terme

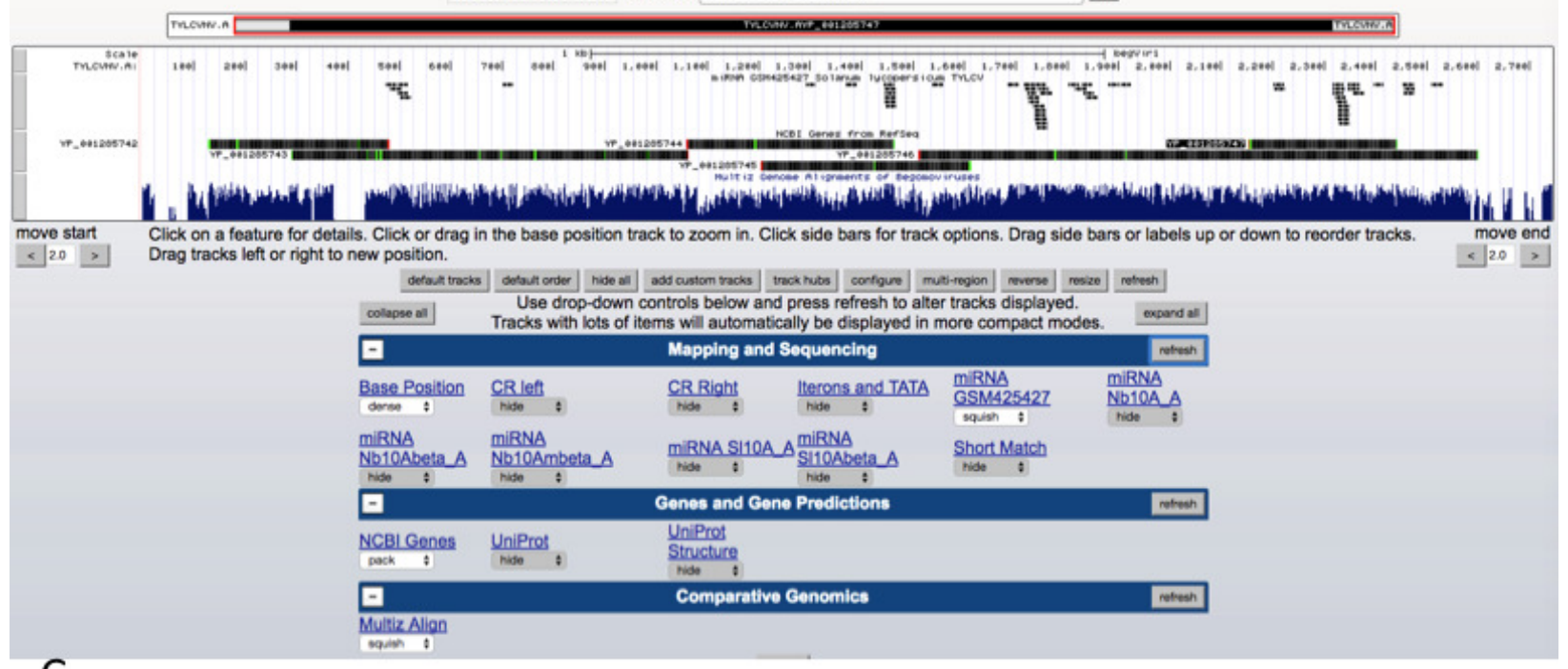

C

NCBI Genes from RefSeq (YP_001285746)

Position: TYLCVNVA:1512-2600

Genomic Size: 1089

Strand: -

Links to sequence:

- Iranslated Protein from predicted mRNA

- Predicted mRNA from genomic sequences

- Genomic Sequence from assembly

\section{Gene Description}

Rep protein of Old World monopartite Tomato yellow leaf curl Vietnam virus DNA-A, complete sequence (TYLCVNV.A) originated from VIETNAM. It was found to infect host "TOMATO (LYCOPERSICON ESCULENTUM, SOLANACEAE); CROP", NC_ 009548 or DQ641697 [Provided by NCBI]

Vew table schema 
330

331

332

333

334

335

336

337

338

339

340

341

342

343

344

345

346

347

348

349

350

351

352

353

354

355

356

357

358

359

Figure 3. Web interface of gb4gv. (A) The home page of gb4gv. The evolutionary tree on the left side of the page shows the available viruses including the three genera of Geminiviruses and two satellites. Users can view a particular genus or satellite by clicking on the virus or satellite name in the evolutionary tree. Users can also make use of the "Species Search" box (above the tree) to look up for a particular virus by keywords. Additionally, users can enter keywords in the "Position/Search Term" box search for a particular virus and/or gene. Click the blue GO button to navigate into the genomic information of a particular viral species. (B) The page at the middle level provides various annotation information about the selected genome in which they are organized in tracks. (C) Information of a protein-coding gene. It tells the genomic location of the gene, size, and strand that codes for the protein. In addition, there is a short description about the current gene including the name of the protein, whether the virus is a New World or an Old World virus, the full name of the virus and its acronym, the RefSeq and GenBank accession numbers with hyperlink linked to the corresponding GenBank entry in NCBI website.

In the following subsections, we will highlight the unique features offered by gb4gv

that are helpful in studying the genomics of geminiviruses. While the software architecture

of gb4gv is based on Genome Browser, the operations of our website is highly similar to

UCSC Genome Browser. Therefore we will not discuss the data models and functionalities

of Genome Browser in details. For readers who are interested in learning more about

Genome Browser, we recommend that they consult the online User Guide (UCSC Genome

Browser User Guide).

Search by Acronym, Accession number, and Attributes

To our best knowledge, there is no database that allows users to search for geminivirus genomes or proteins by acronym, host name, geographical location, monopartite, bipartite, Old world, New world, or combinations thereof. For instance, a search for monopartite begomoviruses that infect Okra by the query "monopartite okra" against NCBI RefSeq database returned only two entries: NC_005954 and NC_005051 and both of them belong to satellite genomes. In fact, four monopartite begomoviruses are known to infect Okra 
360 according to gb4gv: OLCCV (NC_014745), OYCrV (NC_008377), OYVMV (NC_004673), and

361 OkLCuV (NC_013017). The main reason is because NCBI's query matches only words in the

362 description of GenBank entries. Our augmented search capability will help researchers in

363 identifying a regime of viruses that share certain attributes handily. gb4gv achieves this by

364 making the above viral attributes searchable in our database in conjunction with the

365 keyword searching capability provided by Genome Browser. Table 2 below summarizes the 366 searchable attributes supported by gb4gv.

Table 2: Searchable attributes in gb4gv.

\begin{tabular}{|c|c|c|}
\hline Attribute & Description & Example \\
\hline World & $\begin{array}{l}\text { Geminiviruses are commonly categorized into } \\
\text { "Old World" and "New World" according to } \\
\text { the geographical location they were found. } \\
\text { This attribute must be either "Old World" or } \\
\text { "New World" }\end{array}$ & old world \\
\hline Number of main genomes & It must be either monopartite or bipartite & bipartite \\
\hline Acronym of the virus & $\begin{array}{l}\text { For begomoviruses, it could be suffixed } \\
\text { optionally by “.A" or “.B” to indicate DNA-A or } \\
\text { DNA-B of the bipartite genome, respectively. }\end{array}$ & OMOV.A \\
\hline Host & Name of the host infected by the virus & Okra \\
\hline Country & The country that the virus was found & Brazil \\
\hline RefSeq accession number & $\begin{array}{l}\text { The accession number assigned by NCBI } \\
\text { RefSeq database }\end{array}$ & $\mathrm{NC} \_011181$ \\
\hline
\end{tabular}


GenBank accession number The accession number of the GenBank record EU914817 that RefSeq used

369 For instance, to find all begomoviruses that infect sweet potato, user can input the phrase

370 "sweet potato" in the query box and click the "go" button (Figure 4A). 
A

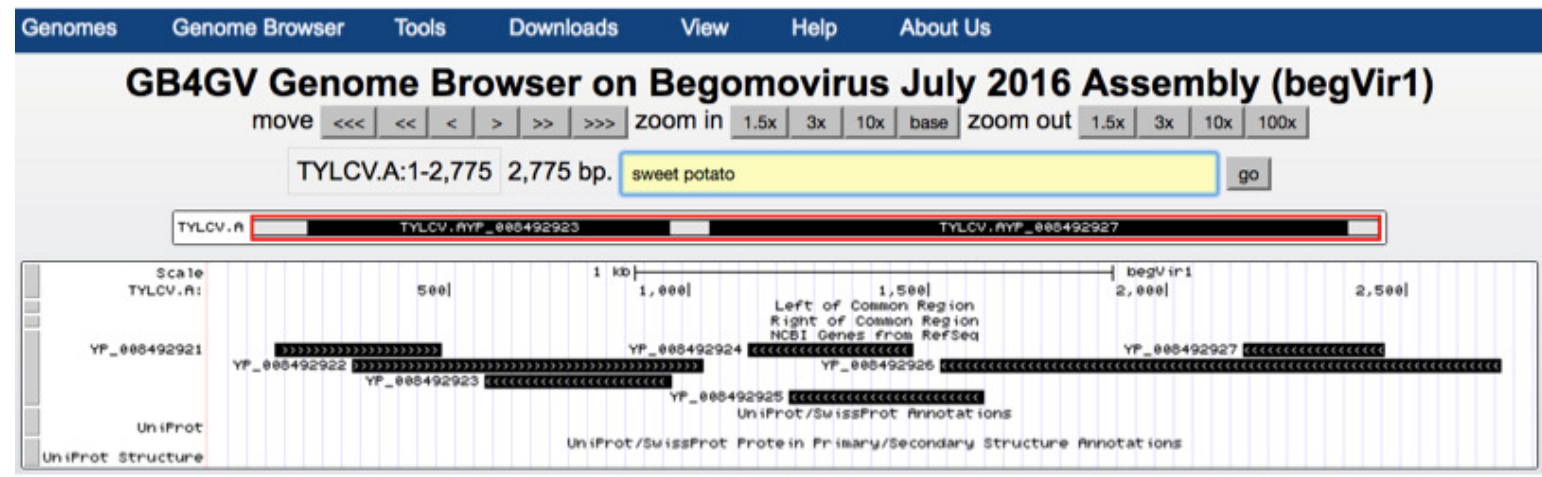

\section{NCBI Genes from RefSeq}

Sweet potato golden vein associated virus, complete genome, at SPGVaV.A:1581-2675 - (YP_004346960) Sweet potato golden vein associated virus, complete genome, at SPGVaV.As:1075-1509 - (YP_004346958) Sweet potato golden vein associated virus, complete genome, at SPGVaV.A:72-470 - (YP_004346956)

Sweet potato leaf curl South Carolina virus, complete genome, at SPLCSCV.Ail526-2620 - (YP_004339041)

Sweet potato leaf curl South Carolina virus, complete genome, at SPLCSCV. A:1017-1454 - (YP_004339039)

Sweet potato leaf curl South Carolina virus, complete genome. at SPLCSCV.A: $90-431$ - (YP_004339037)

Sweet potato leaf curl Uganda virus-[Uganda:Kampala:2008], complete genome, at SPLCUV.A_1544-2638 - (YP_004191799)

Sweet potato leaf curl Uganda virus-[Uganda:Kampala:2008], complete genome. at SPLCUV.A:1038-1472 - (YP_004191797)

Sweet potato leaf curl Uganda virus-[Uganda:Kampala:2008], complete genome, at SPLCUV.A:249-827 - (YP_004191795)

Sweet potato leaf curl Bengal virus - [India:West Bengal:2008] segment A, complete genome. at SPLCV_BCKV.A:2260-2499 - (YP_003560504) Sweet potato leaf curl Bengal virus - [India:West Bengal:2008] segment A, complete genome. at SPLCV_BCKV.A:1222-1671 - (YP_003560502) Sweet potato leaf curl Bengal virus - [India:West Bengal:2008) segment A, complete genome. at SPLCV_BCKV.A:290-1054 - (YP_003560500) Sweet potato leaf curl Lanzarote virus, complete genome, at SPLCLaV.A:2232-2495 - (YP_003288786)

Sweet potato leaf curl Lanzarote virus, complete genome, at SRLCLaV.A:1197-1643 - (YP_003288784) Sweet potato leaf curl Lanzarote virus, complete genome, at SPLCLaV.A:266-1030 - (YP_003288782) Sweet botato leaf curl Canarv virus. comblete denome. at SPLCCanV.A:2231-2488 - (YP 003288774)

B

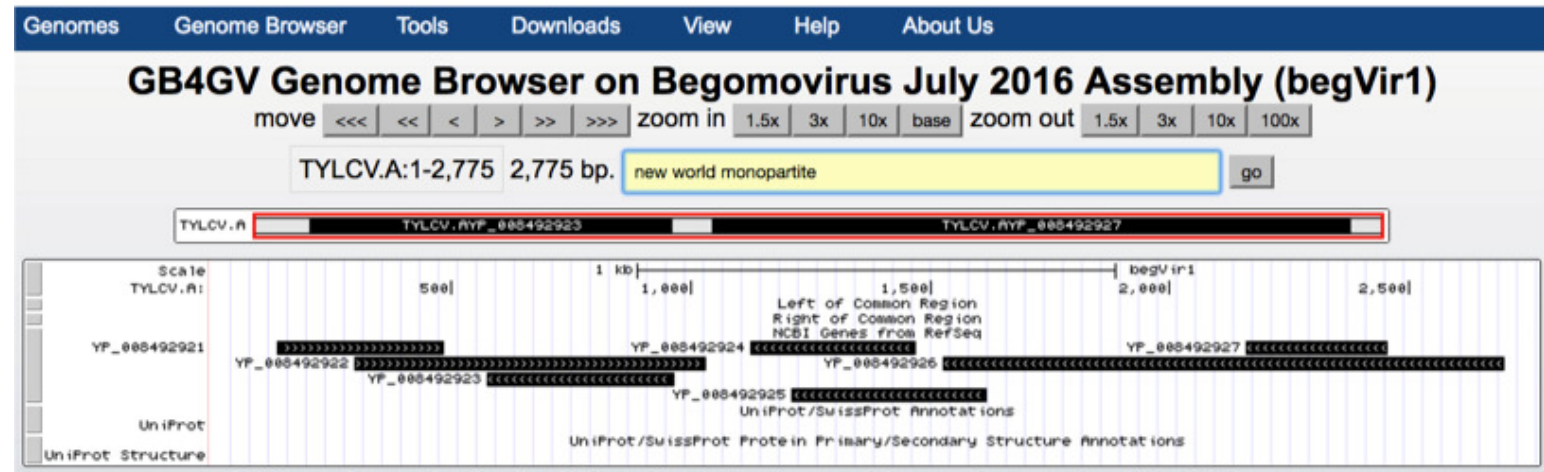

\section{NCBI Genes from RefSeq}

Sida mottle Alagoas virus isolate BR:Vsa2:10 segment DNA-A, complete sequence, at SiMoAV.A:1427-2512 - (YP 007438883) Sida mottle Alagoas virus isolate BR:Vsa2:10 segment DNA-A, complete sequence, at SiMOAV.A:981-1379 - (YP 007438881) Sida yellow mosaic Alagoas virus isolate BR:Vsa3:10 segment DNA-A, complete sequence. at SiYMAV.A:2130-2387 - (YP_007438879) Sida yellow mosaic Alagoas virus isolate BR:Vsa3:10 segment DNA-A, complete sequence. at SiYMAV,A:1158-1547 - (YP 007438877) Sida yellow mosaic Alagoas Virus isolate BR:Vsa3:10 segment DNA-A, complete sequence. at SiYMAV.A:1158-1547 - (YP 007438877 )
Sida yellow mosaic Alagoas virus isolate BR:Vsa3:10 segment DNA-A, complete sequence. at SiYMAV.A:261-1016 - (YP 007438875) Sida yellow blotch virus isolate BR:Rlal: 10 segment DNA-A, complete sequence. at SiYBV.A:1447-2523 - (YP_007438873) Sida yellow blotch virus isolate BR:Rlal:10 segment DNA-A, complete sequence, at SiYBV.A:992-1390 - (YP_007438871) Sida yellow net virus isolate BR:Vic2:10 segment DNA-A, complete sequence. at SiYNV.A:2125-2382 - (YP_007438869) Sida yellow net virus isolate BR:Vic2:10 segment DNA-A, complete sequence, at SiYNV.A:1150-1539 - (YP 007438867) Sida yellow net virus isolate BR:Vic2:10 segment DNA-A, complete sequence, at SiYNV,A:253-1008 - (YP_007438865) Sweet potato golden vein associated virus, complete genome, at SPGVaV.A:1581-2675 - (YP_004346960) Sweet potato golden vein associated virus, complete genome, at SPGVaV.A: 1075-1509 - (YP 004346958) Sweet potato golden vein associated virus, complete genome. at SPGVaV.A:72-470 - (YP_004346956) Sweet potato leaf curl South Carolina virus, complete genome. at SPLCSCV.A:1526-2620- $($ YP_004339041) Sweet potato leaf curl South Carolina virus, complete genome. at SPLCSCV.A:1017-1454 - (YP_004339039) Sweet potato leaf curl South Carolina virus, complete genome. at SPLCSCV.A:90-431 - (YP_004339037)

Figure 4. Keyword search results. (A) Search by host e.g. "sweet potato". (B) Search by a phrase e.g. "new world monopartite". 
375 User can combine multiple search attributes in a query. The logical AND relationship

376 is assumed between attributes. For example, user can enter "new world monopartite" in

377 the search box to search for all New World monopartite begomoviruses (Figure 4B). But

378 the current version of the search function remains primitive as it is virtually inherited from

379 the 'LIKE' search of MySQL, meaning that the order of queried attributes is important.

380 When multiple attributes are specified, they must be arranged according to the order

381 enlisted in Table 2 from top to bottom. For the same example above, the query

382 "monopartite new world" will result in no hits.

383

384 Short Match

385 The ability to support ad-hoc sequence search can help researchers to identify potential

386 short regulatory sequences that can be validated further by experiment. Examples of these

387 regulatory sequences include TATA box (Sanz-Burgos \& Gutierrez 1998), and

388 polyadenylation signal AWTAAA (W means A or T). The Short Match function allows users to

389 search for DNA sequences from 2 to 30 bases with the support of IUPAC ambiguity codes.

390 Figure 5 illustrates how to specify a short sequence match, and how to inspect the context

391 of a hit within a specific region through the Genome Browser's zoom-in function. 
A

Mapping and Sequencing
\[ \begin{array}{c}\text { Short Match } \\ \checkmark \text { hide } \\ \text { cense } \\ \text { squish iedictions } \\ \text { pack } \\ \text { full } \\ \text { uvivu itructure }\end{array} \]

Short Match Track Settings

\section{Perfect Match to Short Sequence}

Display mode: full $\quad$ Submit

Short (2-30 base) sequence: TATAA

B

Genomes Genome Browser Tools Downloads View Help About Us

GB4GV Genome Browser on Mastrevirus July 2016 Assembly (masVir1)

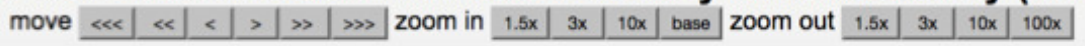

MSV:1-2,689 2,689 bp. enter position or search term

go

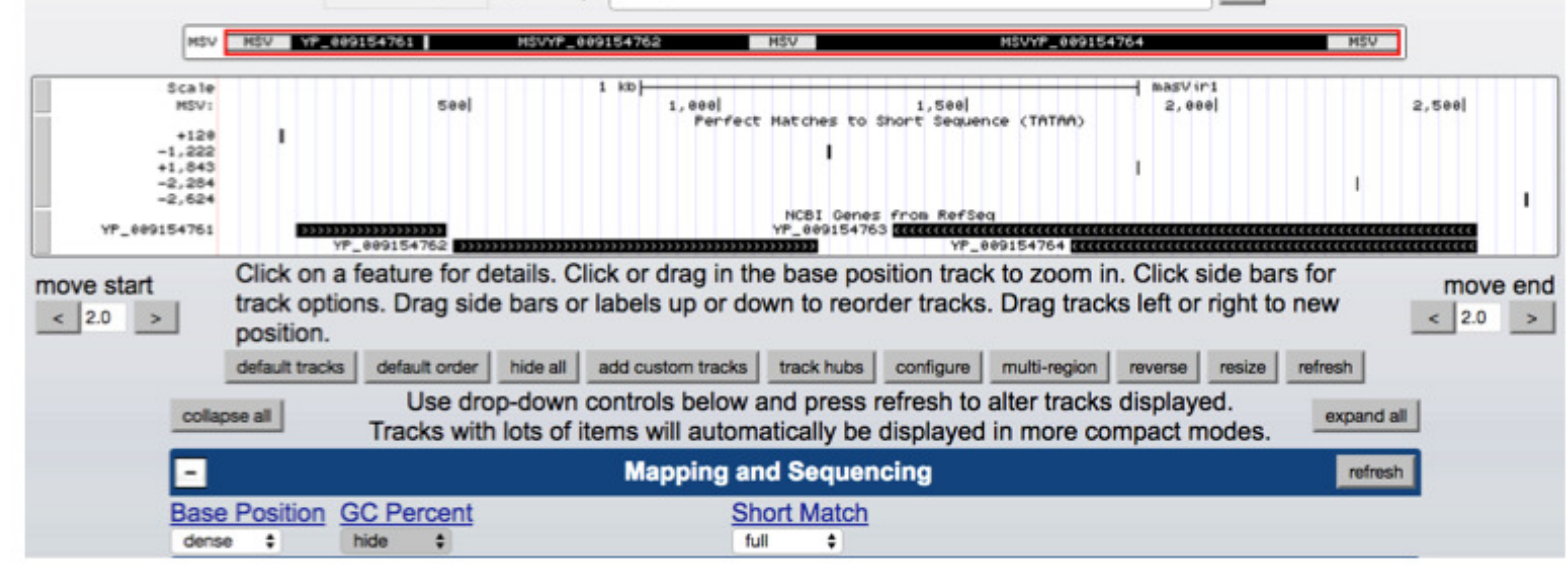

C
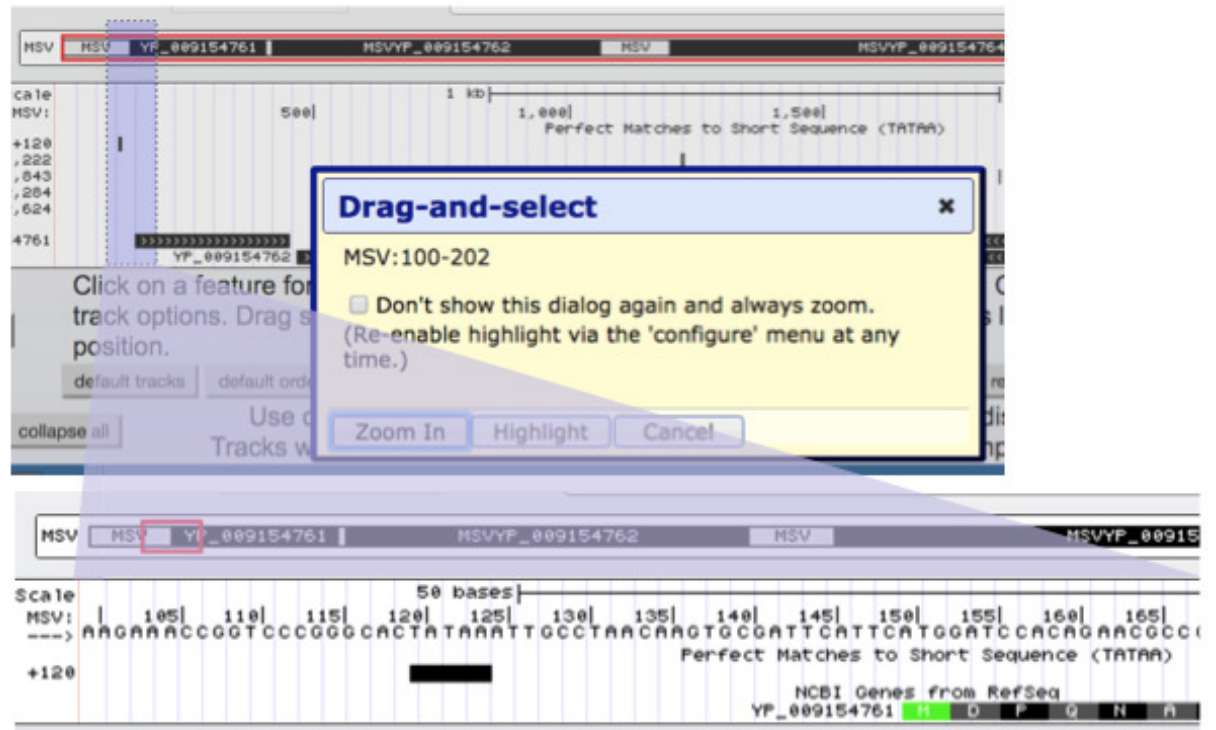

Click on a feature for details. Click or drag in the base position track to zoom i track options. Drag side bars or labels up or down to reorder tracks. Drag trac position. 
393

394

395

396

397

398

399

400

401

402

403

404

405

406

407

408

409

410

411

412

413

414

415

416

417

Figure 5. Setup of Short Match function. (A) Turn on the "Short Match" track to "full", and click the Short Match link. It allows users to input the sequence to search for. (B) After clicked the submit button, Genome Browser will return to the main genome view. If the searched sequence is found, results are displayed under the "Short Match" track including the genomic locations prefixed by a + or - to indicate the hit lies in the reference strand or the complementary strand, respectively. (C) Users can zoom in to a smaller region by dragging the mouse pointer.

\section{Putative Iterons and TATA}

It has been known iterons contributed to viral replication (Arguello-Astorga et al. 1994;

Sanz-Burgos \& Gutierrez 1998). Studied had shown binding activities between REP and iterons in Mastrevirus and begomovirus (Fontes et al. 1992; Sanz-Burgos \& Gutierrez 1998). gb4gv maintains 5,142 putative iterons and TATA box sites in the long intergenic region. Users can view this information by turning on the "Iterons and TATA" track. Figure 6 shows an example of iterons and TATA box sites predicted in begomovirus Melochia yellow mosaic virus (NC_028143).

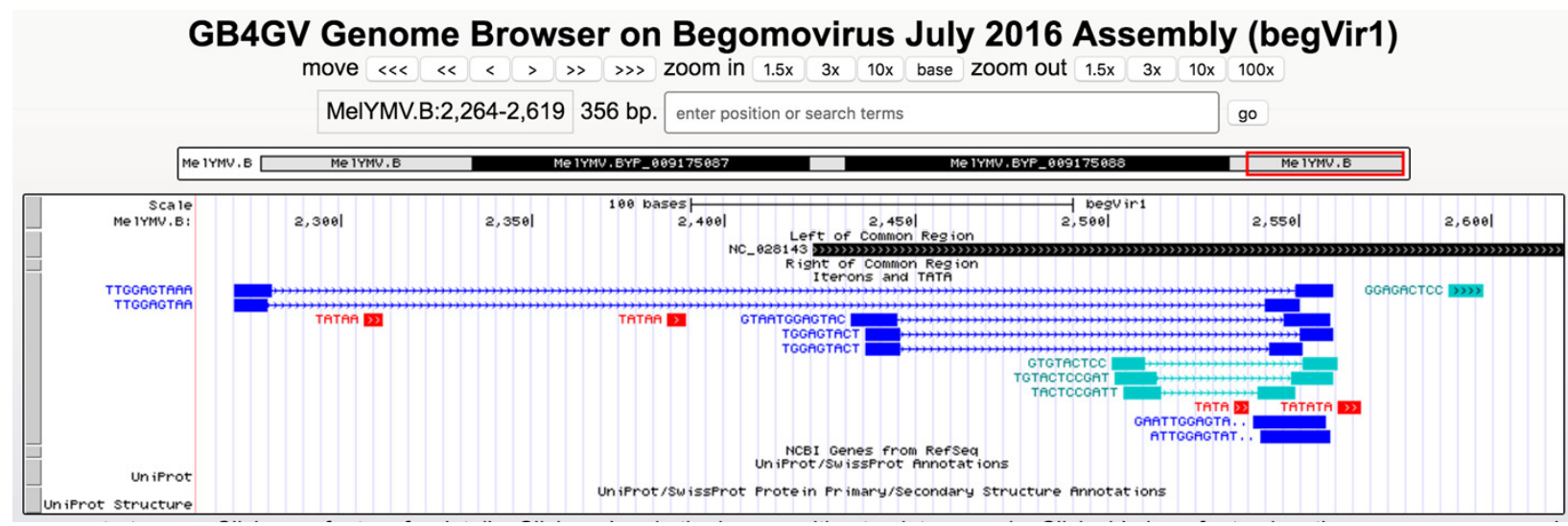

Figure 6. Iterons and TATA track. Different colors are used to denote various sequence features: direct repeats in blue, inverted repeats in blue-green, and TATA box in red. Tandem repeats are highlighted with ".." at the end the label e.g. the direct tandem repeats "GAATTGGAGTA. ." above consists of "GAATTGGAGTATTGGAGTA" in which "GAATTGGAGTA" overlaps with "GaATTGGAGTA" with their overlapping regions underlined. Lastly, our database also highlights palindromic-like sequence by ">>>... > > ", e.g. "GGAGACTCC". 
418 Small Interfering RNAs

419 Understanding plant immunity is the foremost step to fight against viral infection. Virus420 derived RNA silencing is a vital immune response triggered in plants in the face of viral

421 infection. Thus we have incorporated datasets from two virus-derived small interfering

422 RNA (siRNA) studies into gb4gv. One study used pyrosequencing to sequence siRNAs in

423 tomato leaves (Solanum lycopersicum) inoculated with monopartite begomovirus TYLCV

424 (Donaire et al. 2009). Another study had used deep sequencing to survey siRNAs in the

425 leaves of tomato (Solanum lycopersicum) and tobacco (Nicotiana benthamiana) inoculated

426 with monopartite begomovirus and its associated betasatellite (TYLCCNV/TYLCCNB) (Yang

427 et al. 2011). Both studies had mapped the siRNAs to the genomes of respective hosts.

428 However, it is unclear whether or not these siRNA sequences are species specific. Are

429 siRNAs mapped to biased locations? In order to answer these questions, we incorporated

430 siRNA sequences from these two studies into gb4gv and mapped the siRNAs to genomes of

431 begomovirus and betasatellites. Each sample occupies a track (Figure 7A). 


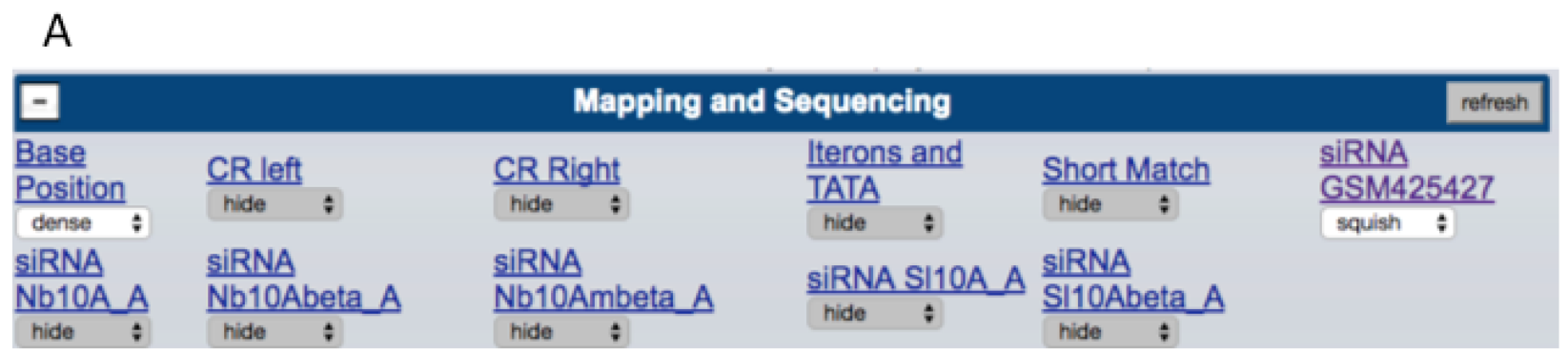

B

GB4GV Genome Browser on Begomovirus July 2016 Assembly (begVir1)

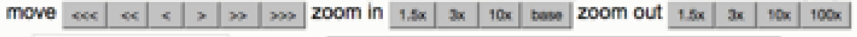
TYLCV.A:463-2,312 1,850 bp. witer pontion or wavech terme $\infty$

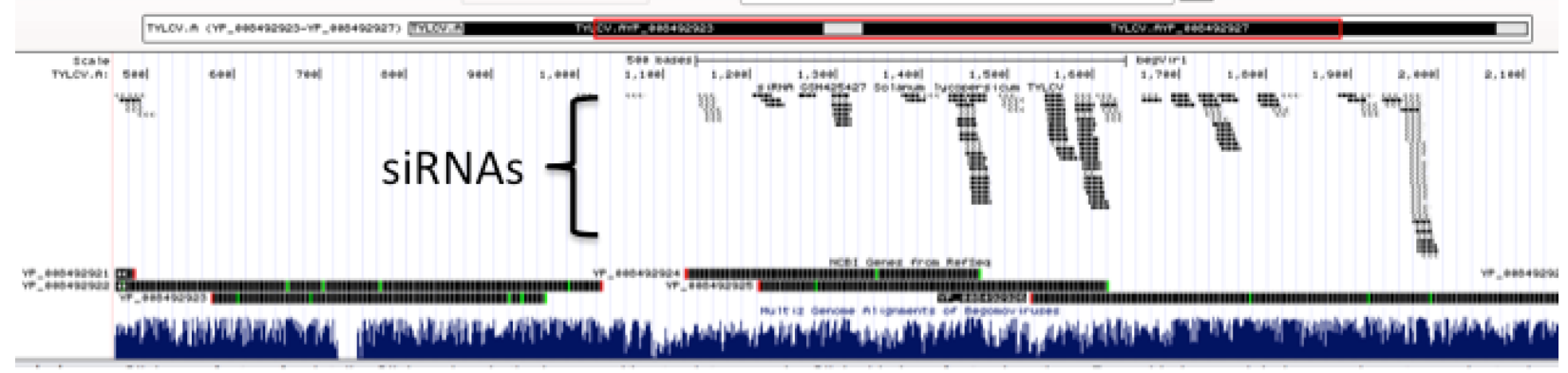

C

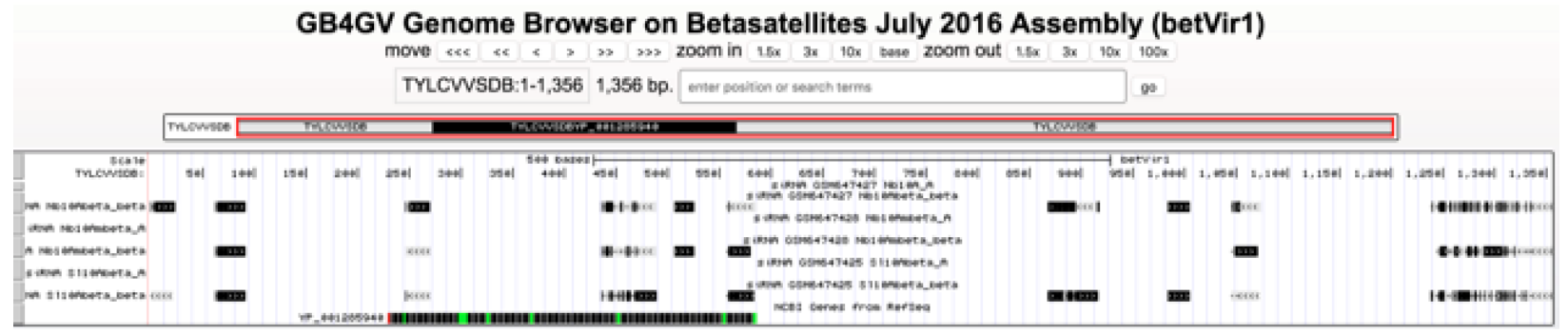

Figure 7. siRNA mapping. (A) Six samples of siRNA sequences are available for from GSM425427 on monopartite begomovirus TYLCV. 'Squish' mode was used in this example. (C) Another example to show the appearance when 'Dense' mode was used to display siRNAs mapped to betasatellite TYLCVVSDB based on samples from GSE26368. and light color, respectively (Figure 7B). According to our limited browsing, siRNAs do not map uniformly along the genome. In betasatellites, a sizeable number of mapped siRNAs were skewed toward a 100-bp region near to the 5' side of the SCE. 


\section{$444 \quad B L A T$}

445 Our database is also equipped with a lightweight sequence query engine BLAT (Kent 2002).

446 BLAT stands for BLAST-like alignment tool. It has been widely used to search for highly

447 similar gapped alignments. In situation like the detection of exons based on a spliced mRNA

448 sequence, BLAT provides a speedy mapping of the query sequence onto the genome. Major

449 differences between BLAT and Short Match are:

450 1. The minimum and maximum query lengths for BLAT are 20 and 25,000 bps,

451 respectively.

452 2. BLAT searches against genomes in a database specified by the user. Whereas Short

453 Match searches for queried sequence only in the current active genome.

454 3. BLAT can handle gapped hit but not for Short Match.

455

456 As an illustration, we used an unusually long (46 bps) iteron sequence

457 “tgagtgattтcttAttAtgtgAttgtcCAttAAAgGgAtAAAgtgA” (Figure 8A) found in

458 YOM (Cotton leaf curl virus betasatellite NC_017829) to query against betasatellite

459 genomes. Intriguingly, eight other betasatellite genomes were found to contain sequences

460 that share from $88.5 \%$ to $97.9 \%$ of identity with the queried sequence (Figure $8 \mathrm{~B}$ ). To

461 further examine the hit in virus LPALDDBV, we clicked the "browser" link on the left, which

462 led to Figure 8C. It shows that the queried sequence hits a region LPALDDBV clustered with

463 iterons. The solid grey bar at the bottom indicates that YOM and LPALDDBV differ at only 464 eight sites.

465 


\section{A}

\section{Betasatellites BLAT Search}

\section{BLAT Search Genome}

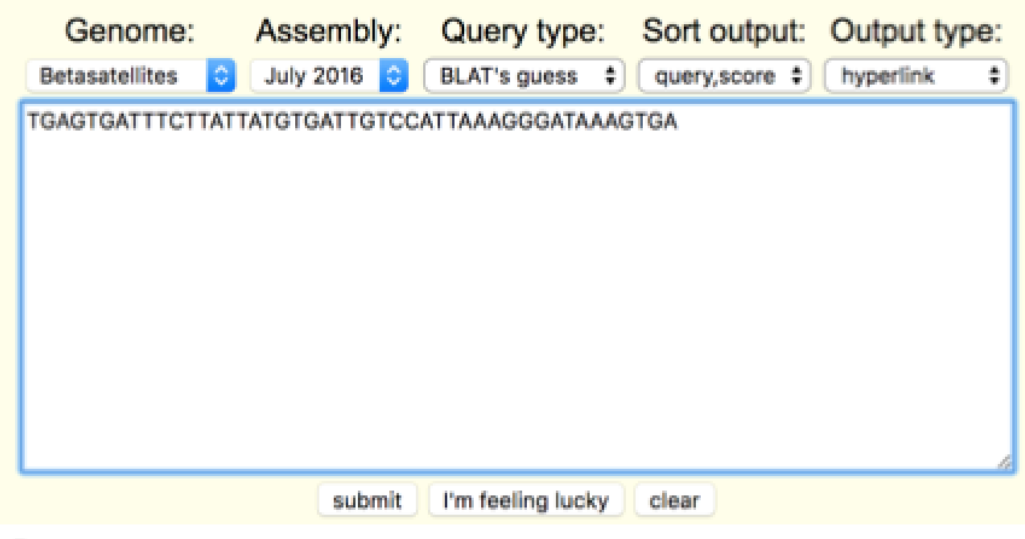

B

Betasatellites BLAT Results

\section{BLAT Search Results}

Go back to ToYL CCCr:1-1339 on the Genome Browser.

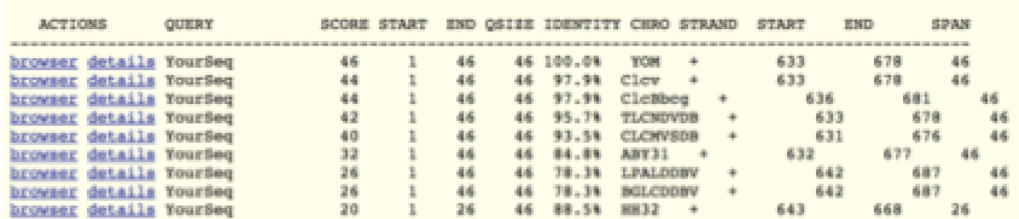

C

GB4GV Genome Browser on Betasatellites July 2016 Assembly (betVir1)

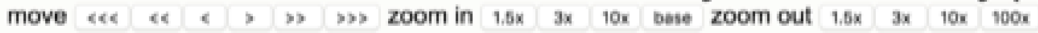

LPALDDBV:631-699 69 bp. enter position or search tems

90

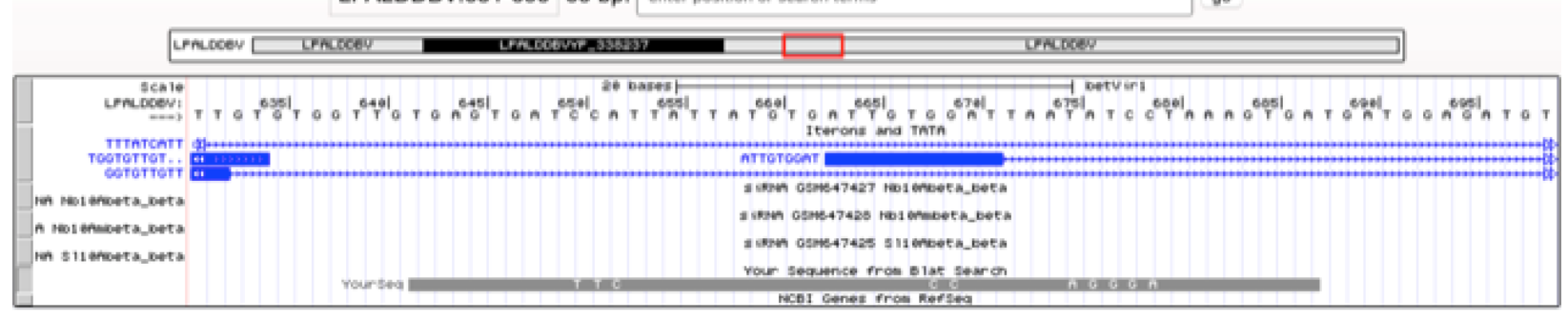

Figure 8. BLAT search. (A) Sequence search box. (B) List of hits with a column of hyperlinks referencing the hit genomes on the far left. (C). Display of hit in the context of targeted genome by clicking the "browser" link on the left of panel (B). The grey bar at the bottom shows the queried sequence is aligned to the opposite strand of the genome. (If the queried sequence aligns to the reference strand, the bar will be displayed in black.) Nucleotides displayed on the grey bar represent mismatched nucleotides between the queried 


\section{Conclusion}

476 Genomics visualization is a useful approach to enhance interpretation especially when the

477 quantity or diversity of the viral genomic data is large. We have harnessed the capability of

478 the widely acclaimed Genome Browser specially for the geminivirus research community.

479 Instead of using a generic one size fits all approach to organize viral genomes, we have

480 taken a semi-automatic pipeline to preserve the unique characteristics of geminiviruses in

481 our web-based database gb4gv. Additionally, we have augmented keyword search

482 capability of manually curated attributes such as infecting hosts, geographical location etc.

483 However, further improvement is needed to accommodate even more flexible multiple

484 attributes queries. Moreover, we have predicted 127 pairs of common regions pertaining to

485 bipartite begomoviruses. This is a useful piece of information as the common regions are

486 implicated in coupling the two main genomes of a bipartite begomovirus during

487 encapsidation. As the ultimate goal in studying the genomes of Geminiviridae family is to

488 understand the underlying genomic features that are suggested to promote its propagation,

489 we have developed our own method to unravel putative iterons and TATA box sites in the

$4905^{\prime}$ side of the common region and they can be visualized readily with genomic features

491 flanking them. The iterons we predicted are longer (9-46 bps) than the iteron core motifs,

$492 \mathrm{GGN}_{1} \mathrm{~N}_{2} \mathrm{~N}_{3}$, reported by this group (Arguello-Astorga \& Ruiz-Medrano 2001). The presence

493 of sizable direct or inverted repeats in fast evolving ssDNA viruses like geminiviruses is

494 unusual, suggesting the existence of negative selective pressure although the biological

495 function of the region peripheral of the iteron core motifs remains largely unknown.

496 Geminiviruses are diverse and fast evolving. Facilitated by the ever-decreasing DNA

497 sequencing cost, we anticipate more viral genomes will be sequenced in the near future. 
498 We are certainly committed to maintaining the information in gb4gv as up-to-date as

499 possible. Given the flexibility of the Genome Browser in accommodating new annotation

500 tracks, if more genome-wide experimental data is available in the future such as Chip-Seq,

501 it can be included into gb4gv readily without software modification as illustrated by the

502 siRNA tracks discussed above. While viral regulatory elements play crucial roles in

503 influencing replication and transcription in cellular environment, we will continue our

504 effort in developing new methods to identify essential sequence elements that might offer

505 new insights for experimental virologists to design effective modalities to fight against the

506 infection of geminiviruses.

\section{Acknowledgements}

508 We thank the UCSC Genome Browser team for their technical support especially to

509 Maximilian Haeussler, Matthew Speir, and Cath Tyner. gb4gv would not be possible

510 without their kind support.

\section{Funding}

512 This project was supported by the startup fund provided by Lafayette College to E.S.H.

513 C.M.N. was supported by the EXCEL Summer Scholar Program funded by Lafayette College.

514 There was no additional external funding received for this study.

\section{References}

516

517

518

519

520

521

522

523
Arguello-Astorga GR, Guevara-Gonzalez RG, Herrera-Estrella LR, and Rivera-Bustamante RF. 1994. Geminivirus replication origins have a group-specific organization of iterative elements: a model for replication. Virology 203:90-100. 10.1006/viro.1994.1458

Arguello-Astorga GR, and Ruiz-Medrano R. 2001. An iteron-related domain is associated to Motif 1 in the replication proteins of geminiviruses: identification of potential interacting amino acid-base pairs by a comparative approach. Arch Virol 146:14651485 . 
524 Bernard V, Brunaud V, and Lecharny A. 2010. TC-motifs at the TATA-box expected position

525

526

527

528

529

530

531

532

533

534

535

536

537

538

539

540

541

542

543

544

545

546

547

548

549

550

551

552

553

554

555

556

557

558

559

560

561

562

563

564

565

566

567

568

569 in plant genes: a novel class of motifs involved in the transcription regulation. $B M C$ Genomics 11:166. 10.1186/1471-2164-11-166

Blanchette M, Kent WJ, Riemer C, Elnitski L, Smit AF, Roskin KM, Baertsch R, Rosenbloom K, Clawson H, Green ED, Haussler D, and Miller W. 2004. Aligning multiple genomic sequences with the threaded blockset aligner. Genome Res 14:708-715. 10.1101/gr.1933104

Briddon RW, Mansoor S, Bedford ID, Pinner MS, Saunders K, Stanley J, Zafar Y, Malik KA, and Markham PG. 2001. Identification of dna components required for induction of cotton leaf curl disease. Virology 285:234-243. 10.1006/viro.2001.0949

Brown JK, Zerbini FM, Navas-Castillo J, Moriones E, Ramos-Sobrinho R, Silva JC, Fiallo-Olive E, Briddon RW, Hernandez-Zepeda C, Idris A, Malathi VG, Martin DP, RiveraBustamante R, Ueda S, and Varsani A. 2015. Revision of Begomovirus taxonomy based on pairwise sequence comparisons. Arch Virol 160:1593-1619. 10.1007/s00705-015-2398-y

Donaire L, Wang Y, Gonzalez-Ibeas D, Mayer KF, Aranda MA, and Llave C. 2009. Deepsequencing of plant viral small RNAs reveals effective and widespread targeting of viral genomes. Virology 392:203-214. 10.1016/j.virol.2009.07.005

Duffy S, Shackelton LA, and Holmes EC. 2008. Rates of evolutionary change in viruses: patterns and determinants. Nat Rev Genet 9:267-276. 10.1038/nrg2323

Edgar RC. 2004. MUSCLE: multiple sequence alignment with high accuracy and high throughput. Nucleic Acids Res 32:1792-1797. 10.1093/nar/gkh340

Felsenstein J. 2005. PHYLIP (Phylogeny Inference Package) version 3.6. Distributed by the author Department of Genome Sciences, University of Washington, Seattle.

Fontes EP, Luckow VA, and Hanley-Bowdoin L. 1992. A geminivirus replication protein is a sequence-specific DNA binding protein. Plant Cell 4:597-608.

GenBank Record. NCBI GenBank Record. Available at https://www.ncbi.nlm.nih.gov/Sitemap/samplerecord.html (accessed 27 December 2016).

Gene Expression Omnibus. NCBI Gene Expression Omnibus. Available at https://www.ncbi.nlm.nih.gov/geo/ (accessed 1 December 2016).

Gutierrez C. 1999. Geminivirus DNA replication. Cell Mol Life Sci 56:313-329.

ICTV. 2015. ICTV. Available at https://talk.ictvonline.org/files/master-species-lists/ (accessed 15 December 2016).

Jeske H. 2009. Geminiviruses. Curr Top Microbiol Immunol 331:185-226.

Jeske H, Lutgemeier M, and Preiss W. 2001. DNA forms indicate rolling circle and recombination-dependent replication of Abutilon mosaic virus. EMBO J 20:61586167. 10.1093/emboj/20.21.6158

Kent WJ. 2002. BLAT--the BLAST-like alignment tool. Genome Res 12:656-664. 10.1101/gr.229202. Article published online before March 2002

Kent WJ, Sugnet CW, Furey TS, Roskin KM, Pringle TH, Zahler AM, and Haussler D. 2002. The human genome browser at UCSC. Genome Res 12:996-1006. 10.1101/gr.229102. Article published online before print in May 2002

Kumar S, Stecher G, and Tamura K. 2016. MEGA7: Molecular Evolutionary Genetics Analysis Version 7.0 for Bigger Datasets. Mol Biol Evol 33:1870-1874. $10.1093 / \mathrm{molbev} / \mathrm{msw} 054$ 
570 NCBI RefSeq. NCBI RefSeq. Available at ftp://ftp.ncbi.nlm.nih.gov/refseq/release/viral/

$571 \quad$ (accessed 15 December 2016).

572 NCBI Viral Genomes. NCBI Viral Genomes. Available at

573 https://www.ncbi.nlm.nih.gov/genome/viruses/ (accessed 1 June 2016).

574 Orozco BM, and Hanley-Bowdoin L. 1996. A DNA structure is required for geminivirus

575 replication origin function. J Virol 70:148-158.

576

577

578

579

580

581

582

583

584

585

586

587

588

589

590

591

592

593

594

595

596

597

598

599

600

601

602

603

604

605

606

607

608

609

610

611

612

613

614

615

Patikoglou GA, Kim JL, Sun L, Yang SH, Kodadek T, and Burley SK. 1999. TATA element recognition by the TATA box-binding protein has been conserved throughout evolution. Genes Dev 13:3217-3230.

Pilartz M, and Jeske H. 2003. Mapping of abutilon mosaic geminivirus minichromosomes. J Virol 77:10808-10818.

Sanz-Burgos AP, and Gutierrez C. 1998. Organization of the cis-acting element required for wheat dwarf geminivirus DNA replication and visualization of a rep protein-DNA complex. Virology 243:119-129. 10.1006/viro.1998.9037

Sattar MN, Kvarnheden A, Saeed M, and Briddon RW. 2013. Cotton leaf curl disease - an emerging threat to cotton production worldwide. J Gen Virol 94:695-710. 10.1099/vir.0.049627-0

Saunders K, Norman A, Gucciardo S, and Stanley J. 2004. The DNA beta satellite component associated with ageratum yellow vein disease encodes an essential pathogenicity protein (betaC1). Virology 324:37-47. 10.1016/j.virol.2004.03.018

Scholthof KB, Adkins S, Czosnek H, Palukaitis P, Jacquot E, Hohn T, Hohn B, Saunders K, Candresse T, Ahlquist P, Hemenway C, and Foster GD. 2011. Top 10 plant viruses in molecular plant pathology. Mol Plant Pathol 12:938-954. 10.1111/j.13643703.2011.00752.x

Shepherd DN, Martin DP, Van Der Walt E, Dent K, Varsani A, and Rybicki EP. 2010. Maize streak virus: an old and complex 'emerging' pathogen. Mol Plant Pathol 11:1-12. 10.1111/j.1364-3703.2009.00568.x

UCSC Admin. UCSC Genome Browser Download. Available at http://hgdownload.soe.ucsc.edu/admin/ (accessed 1 June 2016).

UCSC GB Statistics. UCSC Genome Browser Statistics. Available at http://genome.ucsc.edu/admin/stats/ (accessed 15 December 2016).

UCSC Genome Browser. UCSC Genome Browser. Available at http://genome.ucsc.edu.

UCSC Genome Browser User Guide. UCSC Genome Browser User Guide. Available at https://genome.ucsc.edu/goldenpath/help/hgTracksHelp.html (accessed 15 December 2016).

UniProtKB. UniProt Knowledgebase. Available at http://www.uniprot.org/downloads (accessed 1 June 2016).

Xie Y, Wu P, Liu P, Gong H, and Zhou X. 2010. Characterization of alphasatellites associated with monopartite begomovirus/betasatellite complexes in Yunnan, China. Virol J 7:178. 10.1186/1743-422X-7-178

Yang X, Wang Y, Guo W, Xie Y, Xie Q, Fan L, and Zhou X. 2011. Characterization of small interfering RNAs derived from the geminivirus/betasatellite complex using deep sequencing. PLoS One 6:e16928. 10.1371/journal.pone.0016928

Zhou X, Xie Y, Tao X, Zhang Z, Li Z, and Fauquet CM. 2003. Characterization of DNAbeta associated with begomoviruses in China and evidence for co-evolution with their cognate viral DNA-A. J Gen Virol 84:237-247. 10.1099/vir.0.18608-0 
